\title{
Immersed in cyberspace : an evaluation of a grounded theory approach to a study of user preferences
}

\author{
Alan MacLennan
}

\begin{abstract}
This paper describes a piece of research intended to establish user preferences for the design of virtual worlds for information retrieval, and reflects critically on the methodology adopted. Over 100 students and staff at Robert Gordon University were interviewed, in three cycles of literature review, interview, development and testing. A grounded theory approach was taken, in order to reach conclusions based on user experiences, rather than on the literature. Findings were that preferences appear to be based on affective, rather than structural, criteria, the most influential being familiarity, quality, representation, mediation, etc.

The effectiveness of the grounded theory approach in this piece of research is evaluated, with particular regard to the typical criticisms to which such an approach is subject. It is concluded that there is value in the approach, but the limitations of the approach are acknowledged, and strategies to reduce the effect of these are discussed.
\end{abstract}

\section{Introduction}

A three-dimensional style of interface has been the subject of sporadic development since the early 1990s, as the front end of applications for visualising primarily scientific information on stand-alone machines, and in the field of computer gaming.

The second half of 2006 saw a rise in the usage of virtual reality applications on the World Wide Web, with the widespread popularity of Second Life and Active Worlds, the most successful of the online communities. As of October 2006, Second Life had almost a million accounts; as of May 2011, 1.3 million unique avatars logged in in the previous two months, from over 23 million created, but not cancelled or banned (Second Life, 2011).

\section{Author}

Dr Alan MacLennan is a lecturer and course leader of MSc Information Management at Robert Gordon University, Aberdeen, UK, with interests in programming and virtual realities.

Email: a.maclennan@rgu.ac.uk 
The term "virtual library" has now become quite commonplace, although its denotation is somewhat different from that of "virtual world". A "virtual library" is not typically a representation of a "real world" library, in the same sense that a virtual world might resemble the real one, that is, in giving an illusion of threedimensional space. Instead, virtual libraries, for example the World Wide Web Virtual Library (WWW Virtual Library, 2011) are often more-or-less structured collections of hyperlinks, presented as HTML pages.

Given that so many people are spending time in virtual worlds, and also that the idea of the digital library or virtual library has become well established - a quick web search reveals hundreds of examples - it became interesting to establish what type of interfaces might be favoured for provision of access to information, in these and other "virtual" contexts. Information visualisation techniques developed to enhance retrieval might be considered well suited to the virtual environment, but examples of their usage are comparatively rare. Where virtual reality (VR) or similar techniques have been used in an information retrieval context, there appears to be little evidence that their design has been influenced by user studies, or knowledge of user preferences, and it is this apparent gap in the research which the piece of work was intended to address. The study itself examines the question of what user preferences would be for a virtual world designed to facilitate access to information.

When it became of interest to explore user preferences for a 3-dimensional interface for a world to be used for accessing information, an initial literature search appeared to indicate an absence of user-centred development in this field.

Of the many papers published about digital libraries, in which it might be thought at least the question of information access could be taken for granted, few appear to concern themselves with 3D representations of libraries, and in those, it appears that the decision to use a representation of a library has been taken somewhat arbitrarily.

In a chapter on 'Designing virtual environments', Sutcliffe writes: when the system exists to help the user achieve a task goal, support for the user's task should be explicit. Taking a virtual library as an example, the user's goal is to retrieve specific information and to browse through the library. The application should help the user navigate and locate information, even if this means that the correspondence between the real-world library and its virtual counterpart is violated.

(Sutcliffe, 2003 p.167)

Helpful features might be better signage, improved user guides, or even a complete re-working of the appearance and layout of a conventional library. The range of possibilities is such that it would appear that it could best be narrowed down by consulting potential users. Sutcliffe does note that:

$V R$ has been used in many other applications, including domains where there is little to model, for example, virtual representations of information categories for browsing and retrieval. However, most VR applications have a close correspondence with the real world 
Here, perhaps, is a clue to the scarcity of user studies. If most VR applications are modelled on their real world counterparts, then an information resource maps neatly onto a library, with the possible proviso that it be a helpful environment, which is not necessarily the case with the real thing.

The research discussed here was concerned primarily with the use of 3dimensional worlds in accessing information, and in particular with the questions arising when designing such worlds. If an architect wants to give a virtual tour of a proposed building, or a surgeon wishes to demonstrate a new operating technique, there are fairly obvious factors constraining the choice of an appropriate representation - one must resemble a building, the other some part of a human body. When dealing with a whole range of information, however, there are no such obvious candidates, and indeed it may be that choosing a conventional representation negates the potential benefits of the virtual presentation.

The study aimed to make a contribution to filling the perceived gap in the knowledge regarding user preferences, in that it provided evidence from a usercentred study, which investigated requirements and examined preferences, using interviews and testing interview-derived models. Conclusions drawn from the analysis of this evidence were compared with both the more theoretical material mentioned above, and with actual examples of virtual worlds. This was seen as being a contribution not only to the usability of such interfaces, but also as a valuable set of findings for information providers and libraries who are facing the challenge of providing access to information in such environments.

\section{Aim and objectives}

The aim of the research was to discover user preferences for the design of a virtual world for accessing information, and the factors influencing those preferences.

The objectives of the research were:

- To conduct user interviews, using a grounded theory approach, to elicit user preferences for designs for 3-dimensional "virtual realities" for accessing information.

- To draw from these interviews conclusions as to common elements and re designs.

- To construct "worlds", used to demonstrate different designs as vehicles to develop further depth of understanding of user requirements and preferences.

- To analyse user preferences with particular attention towards affective responses, which might be indicative of the influence of non-structural features of the worlds.

- To draw conclusions as to possible factors influencing user preferences. 


\section{Methodology}

The methodology chosen was reflective of the aim of the research, in that it was intended to discover user preferences as to the design of a $3 \mathrm{D}$ environment for accessing information.

It was decided that the users in the study should have complete freedom in expressing their design preferences. Without such free expression, there is no way of designers knowing reliably what is important to users. This does not mean that designers cannot add functionality to the worlds described by user preferences, because that would tend to stifle development, but that there could be a common understanding of a good basis from which to continue development, and a change in the overall process from design-driven to user-driven.

Preferences were elicited from three groups of users, and were the main focus of the study - they were, as set out in the objectives, used in the design of sample worlds, collected in the form of responses to those worlds, and collected again, with the intention of identifying common factors. It was therefore important to decide on the best methodology for establishing preferences.

\subsection{Naturalistic Inquiry}

Naturalistic Inquiry:

involves studying real-world situations as they unfold naturally in a nonmanipulative, unobtrusive, and non-controlling manner, with openness to whatever emerges and a lack of predetermined constraints on outcomes. The point is to understand naturally occurring phenomena in their naturally occurring states

(Linton, Joy et al, 1999 p.132)

The distinction between this and the ethnographic model is that the study is of real world experiences, but not everyday ones. Lincoln and Guba write:

[t]he human instrument builds upon his or her tacit knowledge as much as if not more than upon propositional knowledge, and uses methods that are appropriate to humanly implemented inquiry: interviews, observations, unobtrusive clues, and the like. Once in the field, the inquiry takes the form of successive iterations of four elements: purposive sampling, inductive analysis of the data obtained from the sample, development of grounded theory based on the inductive analysis, and projection of the next steps in a constantly emergent design. The iterations are repeated as often as necessary until redundancy is achieved, the theory is stabilized, and the emergent design fulfilled to the extent possible in view of time and resource constraints.

[emphasis in original] (Lincoln and Guba, 1985 p.187)

There is also another important step:

Throughout the inquiry, but especially near the end, the data and interpretations are continuously checked with respondents who have acted as sources... differences of opinion are negotiated until the outcomes are agreed upon or minority opinions are well understood and reflected. 
(Lincoln and Guba, 1985 p. 188)

This is the model found to be appropriate to the research discussed here, because the iterative structure provides the opportunity for refining and testing of theory based on findings which, being of a qualitative and essentially subjective and personal nature, require exploration and reformulation before they can be used to advance the theory. The checking with respondents not only confirms understanding of the original content, but also provides an opportunity for respondents to agree with, or dispute, the eventual findings. This "doublechecking" aspect of the inquiry is seen as desirable, in that it affirms the integrity of the process as a whole.

\subsection{Development of the methodology}

\subsubsection{Grounded theory}

The problem centred on determining user preferences without unduly influencing their responses. It was also thought possible that user preferences would turn out to be a matter of personal taste, or dependent on some then-unknown factor or factors. For this reason, a simple statistical representation of user selection from a limited list of arbitrary models was felt to offer an inadequate picture of what a number of individuals would actually want. There was a danger of the findings becoming biased towards a limited choice of models, and that the very selection of these models would be researcher-led. Also, the literature was inadequate to permit development of the range of themes which would allow construction of instruments such as questionnaires or semi-structured interviews.

For these reasons, a decision was made to use a methodology known as Grounded Theory (Glaser and Strauss, 1967). The central concept of Grounded Theory is that theory should "emerge from" data. Glaser and Strauss worked in the field of sociology, and were critical of the approach to research prevalent at the time, where the research process consisted of the development of hypotheses and the subsequent testing of these hypotheses by gathering of data. "Verification of theory is the keynote of sociology" (Glaser and Strauss, 1967 p. 10) This approach led to the development of "great man", or "grand" theories, which were put forward by leaders in the field, and which later sociologists were encouraged to reformulate and test, but without generating theories of their own. Glaser and Strauss' point was that some of the "grand" theories were not grounded in data, and so "do not fit, or do not work, or are not sufficiently understandable to be used". (Glaser and Strauss, 1967 p. 11) However, the generation of theory was not in itself difficult, and techniques could be developed which would permit generation of theories grounded in data, rather than verification of hypotheses an inductive, rather than a deductive, methodology. The authors acknowledged the importance of the uses of both quantitative and qualitative data in both the generation and verification of theory, and denied that the two were incompatible, asserting rather that their importance depended on the requirements of the research and the researcher at the time. They also held that it was frequently the case that both kinds of data were needed, in order to test each other, and to complement each other in the generation of theory. 
The methodology Glaser and Strauss set out for the generation of grounded theories uses comparative analysis, the units of comparison ranging in scale from individuals through organisations to countries. Evidence relating to the research area is gathered, and "conceptual categories" emerge from this evidence. These conceptual categories are somewhat akin to the facets in a faceted classification scheme - they are groupings, or categories, of properties or values related to a concept. The conceptual categories then become entities which can be developed and explored by further examination of the evidence from which they have emerged: "the evidence from which the category emerged is used to illustrate the concept" (Glaser and Strauss, 1967 p. 23).

The types of theory which can emerge from comparative analysis are described as "substantive" or "formal" - the former developed for an "empirical area of sociological enquiry", the latter for a "conceptual area of sociological enquiry". The former is concerned with actual events and processes - the authors give "patient care" as an example. Formal theories are at a greater level of abstraction from the empirical - "authority and power" is an example used.

With the focus on a substantive area ...the generation of theory can be achieved by a comparative analysis between or among groups within the same substantive area.

(Glaser and Strauss, 1967 p. 33)

An emphasis is placed on the use of substantive theory in the generation of grounded formal theory, rather than on the search for an existing formal theory which might have application in a particular substantive area. This "bottom-up" approach was used in the study - development of higher level theories would only be meaningful in the light of further, related, research.

"Incidents" in the data are coded into as many categories as possible. The term "incident" appears to be applicable to an event, or to a reference in an interview, or even to a datum taken from literature. As with much of the writing of Glaser and Strauss, their sociological orientation makes the sense of the text rather obscure, but Strauss and Corbin express this more clearly:

[o]nce we have identified particular phenomena in data, we can begin to group our concepts around them... The process of grouping concepts that seem to pertain to the same phenomena is called categorizing.

[emphasis in original] (Strauss and Corbin, 1990 p. 65)

Categories can be named by the researcher, or can be taken from technical literature. In the case of the research, incidents and categories were drawn from, and related very closely to, user responses to interactions with the worlds.

The next step in the generation of grounded theory is the emergence of hypotheses relating the categories, which together with the categories form "an integrated central theoretical framework - the core of the emerging theory." [emphasis in original] (Glaser and Strauss, 1967 p. 40) The theory is further developed by a process called "theoretical sampling" - a deliberate attempt to collect data which is relevant to the emerging theory, by targeting groups and using methods most 
likely to produce the required data. The study adopted this approach in the selection of groups for each "round" of the process.

Strauss notes that the research question in a grounded theory study may be chosen because:

there is the assumption that someone has never asked this particular research question in quite the same way, so it is as yet impossible to determine which variables pertain to this area and which do not.

(Strauss and Corbin, 1990 p. 37)

In the study, there did not appear to be sufficient data in the literature to indicate that the research question had been asked at all, and this assumption therefore appeared warranted. Indeed, as indicated above, the lack of literature was such that this particular way of conducting the research seemed the only one appropriate. There was no reliable way to choose variables to examine using more structured instruments.

Literature "enables the user to identify previous research in an area, as well as to discover where there are gaps in understanding" (Strauss and Corbin 1990 p. 49).

[T] here is no need to review all the literature beforehand... because if we are effective in our analysis, then new categories will emerge that that neither we, nor anyone else, had thought about previously... It is only after a category has emerged as pertinent that we might want to go back to the technical literature to determine if this category is there, and if so what other researchers have said about it.

(Strauss and Corbin, 1990 p. 50)

The literature was used at stages throughout the research, in order to examine whether other researchers had written anything relevant in related areas, such as information visualisation, for example. The findings influenced the literature which was reviewed at any particular stage of the study, so that the review developed in parallel with, but antecedent to, the interview process.

\subsubsection{The schism in Grounded Theory}

It should be noted here that the discussion above uses points from both Glaser and Strauss' early work, 'The discovery of grounded theory : strategies for qualitative research' Glaser and Strauss, 1967), and Strauss and Corbin's version of Grounded Theory, published in 'Basics of qualitative research : grounded theory procedures and techniques' (Strauss and Corbin, 1990) Glaser and Strauss eventually came to hold rather different positions on what the "true" version of grounded theory should be. There appear to have been two major areas of eventual disagreement between the two approaches, regarding literature and coding procedures, but although both approaches were investigated at length, neither area of disagreement was found to be of relevance to the application of grounded theory in the study.

There is an ongoing debate as to the definitive version of grounded theory, one of the most prolific contributors being Kathy Charmaz. "Glaser and Strauss talk about theory as emerging from data separate from the scientific observer" 
(Charmaz, 2006 p.10). Charmaz, though, says that the theories are interpretations of the world being studied, rather than being exact descriptions. What participants say and mean, and the theories which the researchers reach, are constructions. Glaser (2002) says that "All is data", but Charmaz (2006) points out that data are constructed by people. Glaser (1998) is not too concerned about limited data, because grounded theory develops categories, and data collection can be targeted to develop and explore those categories. Charmaz (2006) says that is a "smash and grab" approach, leading to "superficial analyses".

Glaser (2009) denies assertions in Bryant and Charmaz's (2007) book that he has moved away from discovering basic social process to elucidate the phenomena being researched (he says they are "only one type of theoretical code"), and he dismisses their work as jargonizing QDA (quantitative data analysis), the implication being that the terms used by grounded theory have been appropriated by Bryant and Charmaz's version of grounded theory, to a purpose which is not actually grounded theory.

There are many other contributors to the debate, Glaser's views being propounded on the web site of the Grounded Theory Institute (2012), "dedicated to helping people learn about authentic Grounded Theory (otherwise known as Glaserian, Classic, or Orthodox Grounded Theory)." These claims and counter-claims make it difficult to say authoritatively that a certain theory is grounded, though the techniques used have originated in those described by Glaser and Strauss (1967).

\subsubsection{Criticisms of Grounded Theory}

Bryman (1988) notes three widely-acknowledged problems in qualitative research. First is that of interpretation - "how is it feasible to perceive as others perceive?" (Bryman, 1988 p. 73) How can the researcher have the same perspective as the subject? Even an air of detachment and pure reporting does not reproduce the subjects' viewpoint. The researcher's interest may not be part of the subjects' concerns. The question is:

whether researchers really can provide accounts from the perspective of those whom they study, and how we can evaluate the validity of their interpretations of those perspectives

(Bryman, 1988 p. 74)

In the context of ethnography, one possible means of remedying this situation is to supply field notes and extensive transcripts, in order that readers can draw their own conclusions and reach their own interpretations of the data. There is also the approach of "constitutive ethnography" (Bryman, 1988 p. 78) which additionally seeks to "preserve the social world that is being investigated as data for others to interpret" (Bryman, 1988 p. 78). Respondent validation is another approach to solving this problem, but the respondents are not necessarily validating the data as presented in translation for an academic audience - "It is unlikely that respondent validation will greatly facilitate the ethnographer's second-order interpretation of subjects' first-order interpretations" (Bryman, 1988 p. 79). In fact, respondent validation can be useful input to the second of the three stages of presenting the respondent's world view: the view itself, the researcher's interpretation of that 
view, and the researcher's construction of an interpretation for the academic audience.

These three basic ingredients are inherent in any attempt to provide an interpretation of other people's interpretations for a social scientific audience.

(Bryman, 1988 p.81)

The implication is that checking is limited in its usefulness to an affirmation that the researcher has transcribed accurately, and has not misrepresented the actual content of the data that was collected. However, whilst the three ingredients must be considered carefully, these are considerations of greater import for an ethnographic study, and it has been discussed in section 2.1 why this is not considered to be such a study.

The second question relates to whether research can be conducted in a theoryneutral way, and with specific regard to Grounded Theory, whether it actually provides theories, or simply generates categories. There is a question on a practical level as to whether theory can actually be generated during data collection, especially given the effort entailed in recording, transcribing, coding, etc, or whether it is, in fact, generated afterwards. In the present research, it would be true to say that the theory, substantive rather than formal, was arrived at during the transcription, rather than the collection phase, although the direction of the collection phase had been influenced by the development of the theory.

Another aspect of the theory-generating issue which Bryman identifies is that researchers may be reluctant to depart from what they get from the research, and introduce theoretical elements.

In addition to the emphasis on naturalism... is the predilection for contextualist understanding... This tendency inhibits comparison with other contexts and thereby discourages theoretical development.

(Bryman, 1988 p. 86)

This point, again, appears more directed towards ethnographic studies, particularly of "deviant groups and their subcultures" (Bryman, 1988 p. 85), rather than the participants in the study, who varied as to culture, ethnicity, age, life experience, and in many other attributes.

The third question is whether theory based on a study in a single setting, of a particular case, or of a particular group, can be generalised outside that setting. Bryman considers possible solutions: the study of more than one case, the involvement of teams of researchers, and the selection of either "typical" or "deviant" cases. All of these strategies, though, have their own problems relating to generalisability. Bryman suggests, however, that these perceived problems arise from a misunderstanding of the aims of case study research - that, as in this case, a wide range of individuals is studied, and that the generalisation which takes place is from cases to theories, rather than to larger populations. "Case study data become important when the researcher seeks to integrate them with a theoretical context" (Bryman, 1988 p.90). The grounded theory approach, he writes, “exemplifies this reasoning: a 'substantive theory' ... is then translated into a formal hypothesis". In Glaser and Strauss' work, the substantive theory is about 
the social loss of dying patients, the formal hypothesis is about the social value of the individual related to access to services.

Rather than attempting to defend the questionable position that the (interpreted) experience of a specific group can be generalised to a larger population, as a quantitative survey might generalise quantifiable data about a rigorously sampled group of participants, grounded theory encourages the generation of formal hypotheses, which are open to testing against other contexts.

Sperber and Wilson (1995) present a theory of communication which could be seen as problematic for Grounded Theory. They claim that communication can be described as having an inferential model, that:

an act of ostention carries a guarantee of relevance, and that this fact-which we call the principle of relevance - makes manifest the intention behind the ostention

[emphasis in original] (Sperber and Wilson, 1995 p.50)

Part of an act of communication lies in making it clear that the intention is to communicate and that the content of the communication is, as it were, vouched for as being relevant to the audience. A potential difficulty for the Grounded Theory practitioner is that the authors allow for the possibility of unintended ostensive communication, but add "It would be easy ... to ... make intentionality a defining feature of communication." (Sperber and Wilson, 1995 p. 64) If intentionality were a defining feature, then it becomes difficult to justify the interpretive act of deriving from interviews meanings which are not those expressed directly by the interviewees.

Grounded theory has been criticised for its failure to acknowledge implicit theories which guide work at an early stage. It is also clearer about the generation of theories than about their test. Used unintelligently, it can also degenerate into a fairly empty building of categories (aided by the computer software programs already discussed) or into a mere smokescreen used to legitimise purely empiricist research

(Silverman, 2006 p. 96)

Silverman also claims that, amongst other "cookbook means" of resolving technical issues to which analytical questions are reduced, "simplistic versions of grounded theory ... are no substitute for theoretically inspired reasoning" (Silverman, 2006 pp. $386-7$ ).

\subsection{Selection of users}

Glaser and Strauss write:

[t] he basic criterion governing the selection of comparison groups for discovering theory is their theoretical relevance for furthering the development of emerging categories. The researcher chooses any groups that will help generate, to the fullest extent, as many properties of the categories as possible and that will help relate categories to each other and to their properties. 
[emphasis in original] (Glaser and Strauss, 1967 p.49)

The user groups selected for the first and second rounds of interviews were composed of postgraduate students on Information Management courses at the Aberdeen Business School, Robert Gordon University, Aberdeen. The principal reason for this is that it gave a constituency who were already familiar with the concept and practice of accessing information, and thereby rendered unnecessary the scene setting and preparatory orientation which, in the case of this sample, had already been accomplished through experience of practical retrieval problems. There could be some degree of confidence that interviewer and interviewees were communicating in a common universe of discourse.

The user group for the third round of interviews was composed of academic and technical staff, also from the Aberdeen Business School. This decision was taken partly for the same reasons as applied to the selection of the first two groups, but also as a theoretical sampling decision, in keeping with grounded theory principles. In addition to having the familiarity with accessing information which made the students suitable subjects, it was felt that the staff typically would have greater experience, and would be more practised communicators. This was borne out by the subsequent findings of the study.

\subsubsection{Formative nature of sample}

The sampling was therefore formative - there was a basic requirement that the interviewees were to some extent familiar with the idea of accessing information, but beyond this, the reason behind the sampling was that the tacit knowledge in each of the groups could be made to play its part in the progress of the research. The students came from a wide range of previous academic backgrounds, and brought a correspondingly wide range of experience and viewpoint. At the first stage, the intention was to elicit a range of tacit knowledge regarding information access from people who had not necessarily considered the possibilities of using a $3 \mathrm{D}$ world for this purpose.

The second round of interviews, with a similar group of students, was again intended to elicit tacit knowledge, this time in reaction to sample worlds created in response to the first round of interviews. The reactions of the interviewees were then fed into the next stage of model development.

The staff comprising the third group, many of whom are research-active, again brought a wide range of personal and academic backgrounds, added to greater experience of information access, applied to particular subject areas. This greater practical and theoretical knowledge, combined with greater experience in expressing themselves, was considered to constitute the best group for the final stage of the research, in which the individuals with this body of knowledge, shown the examples derived from the first two groups of interviews, could extrapolate to an "ideal world" for each.

\subsubsection{The interviewees}

All 101 interviewees in the study were volunteers, and were assured anonymity. The size of the sample was determined largely by who volunteered, and the 
sample was therefore to some degree self-selecting, as such samples, without the use of some form of incentive or coercion, will tend to be. This is perceived as a positive feature - those who volunteer are those who are interested in contributing, and the drive behind the research is to go some way to filling the gap in the literature where it appears that user consultation has been lacking. There are still some interviewees who, whilst willing to help, did not respond to the notion of $3 \mathrm{D}$ virtual worlds, and this is to be expected, as the first round of interviews demonstrated that a "one size fits all" solution is unlikely. Some people simply do not find with this type of interface usable, some find their working practices unsuited for reasons of perceived speed and efficiency, some may perceive the interface as somehow frivolous, or better suited to gaming than to work.

\subsection{Selection of interviews as an instrument}

The decision to use interviews as a research instrument was taken because of the nature of the information sought. Since it had been decided that a choice amongst arbitrarily-selected models was not a satisfactory way of establishing what the users really wanted, in a situation where almost any imaginable model could be implemented if desired, and since there was a wish not to influence the choice by presenting a selection of models by which interviewees might be influenced in their choice, it seemed inappropriate to administer a questionnaire. In any case, the fact that the idea of accessing information through a 3D environment had been a novel one to the group in the earlier study, and was relatively uncommon outside the literature on information visualisation, led to the conclusion that a more flexible, personal, contact would be valuable in explaining what might be an unfamiliar idea, and providing reassurance, where necessary. Development of details of interviewing technique also took place in a reflective manner throughout the interviewing stages.

Interviews were very loosely structured. Glaser says:

If the data is garnered through an interview guide that forces and feeds interviewee responses then it is constructed to a degree by interviewer imposed interactive bias. But... with the passive, non structured interviewing or listening of the GT interview-observation method, constructivism is held to a minimum.

(Glaser, 2002 paragraph 11)

He further defends against an accusation of constructivism (the idea that findings are unduly influenced by the interviewer):

Let us be clear, researchers are human beings and therefore must to some degree reify data in trying to symbolize it in collecting, reporting and coding the data. In doing so they may impart their personal bias and/or interpretations-ergo this is called constructivist data. But this data is rendered objective to a high degree by most research methods and GT in particular by looking at many cases of the same phenomenon, when jointly collecting and coding data, to correct for bias and to make the data objective.

(Glaser, 2002 paragraph 28) 
Interviewees were encouraged to talk freely, even when this led to lengthy digressions. Interviewer contributions were limited, as far as possible, to prompting interviewees when "stuck", or bringing them back to the main topic.

Interviews were tape-recorded with the permission of the interviewees, and transcribed, interviews being assigned a number at the transcription stage. It was felt that tape recording was a relatively non-intrusive way of recording interviews, and all interviewees agreed to it without reservation. Interview transcripts were subsequently checked for accuracy with the interviewees, revealing one minor misunderstanding in one interview, which was corrected accordingly. This "member-checking" is in accordance with the procedures recommended by Lincoln and Guba (1985 p. 188).

\subsection{Iteration}

Although the continuing availability of the same subjects over the course of the research would have been problematic if an extended quantitative study had been the methodology of choice, the grounded approach meant that there was no particular requirement to interview the same individuals several times - just to interview individuals, although sessions were therefore slightly longer, to allow for scene-setting. As long as the necessary information was acquired, there was no necessity to repeat interviews. The development of the theory, and of the research instrument itself, takes place independently of any development in knowledge or skill on the part of the interviewee.

For this reason, the methodology, like the literature review, was treated in a sectional, or sequential, manner. Grounded Theory allows, and indeed expects, that the theoretical structure will be developed through rounds of, in this case, interviews, and that each round will be both founded on previous rounds, and an attempt to reflectively develop a research instrument of greater precision than in the previous round.

\section{Conclusions}

This study approached the question of designing virtual worlds from a usercentred perspective, which appears to have been missing from other treatments of the subject. It found that it is possible to derive from interviews a set of properties which are distinct from, and complementary to, those considered in other publications. For example, it is widely acknowledged that "intelligibility" is a positive factor in the design of 3D worlds, but it does not appear to have been considered that familiarity of an environment might also play a significant part in the acceptance of the world as a place in which to work.

It was found that the properties of familiarity, organisation or structure, mediation or assistance, and quality of presentation were those deemed to be important by the participants in this study. It is felt probable that these properties, rather than the tendency to select a particular design, will be transferable across different groups of users, and that these findings can help to determine the course of further research and design work in the area of $3 \mathrm{D}$ worlds for information retrieval. 
Faced with the problem of organising information resources in a 3D virtual environment, the person will fall back on a familiar setting, which enables them to interact with the resources in a way with which they are at ease. In Grounded Theory terminology, the phenomenon is the expression of preferences, the context is the array of resources, and the conditions are the feelings of uncertainty, unfamiliarity, and so on. The consequences would be that they get a world they can deal with. The advice to developers would be: Do not attempt to create anything too elaborate or abstract to begin with - aim for the familiar, but leave scope for personalisation and customisation, because users will soon want to tailor the world to their emerging preferences.

It appears that familiarity is just as important as structure, so in a world of one's own design, both these factors can be covered. In a designed world, there would have to be either a balance, or enough of one to compensate for a lack of the other.

There is a desire for clear organisation, either by a classification scheme, as in a library, more loosely by topic, as in shops, or by a personal arrangement.

People often appear to want their information to be mediated. It is part of the metaphor in both libraries and shops that a selection based on quality has been made, and it is a common criticism of the internet that no quality control of information is possible.

The other important aspect of mediation is assistance. Again, this is not a feature required by everyone, but there is a definite demand from some. While this phenomenon may have been perceived as symptomatic of a lack of training, or simple inexperience, setting it in the context of professional mediation shows it in a different light. A successful library user will know when to ask a librarian for assistance; a successful shopper will call on the skills of the staff. The fact that people will choose a virtual environment with this feature "built in" gives us a significant insight into their relationship with the world of information.

Even when a choice was made from a limited range of models, the grounded approach revealed a widespread desire for customisability of the particular model chosen, and also a readiness to extend the model. For example, worlds can be enhanced by providing the ability to leave a trail, or a library can be enhanced by having the sought items move towards the user.

Finally, there is the issue of quality. In the same way that people require quality of information, they also want a high-quality interface. A "cheap and cheerful" version may be usable, but, in addition to a more aesthetically pleasing experience, there is also the potential to offer enhanced functionality by exploiting latent skills. The abilities to remember where one put something, or to observe wear and tear caused by use may operate at almost subconscious levels, but can be used to add value to the experience of accessing information.

It is thought that this research makes a contribution in the field of information behaviour, in that it has identified non-structural influences which can affect individuals' interaction with interfaces for accessing information. Although this type of research is time-consuming, it has led to insights less likely to be achieved by other methodologies. 
There is also a contribution in the field of information systems design, in that it appears that a "softer" approach, which is more sensitive to properties other than the simply structural, may have unforeseen benefits to the design process. The conclusion that users may not be as concerned with the actual model used for the 3D world, as they are with its familiarity, or the fact that they can recognise its organisation, or get dependable assistance, is the result of a different approach to those taken before, and merits further investigation.

\section{Evaluation of research approach}

The research approach adopted was that of Naturalistic Inquiry. As explained in section 2.1.3, this iterative methodology is intended for examining real world situations, but not ones which are necessarily "everyday". The iterative approach allowed each cycle of interviews, analysis, literature review and discussion of emergent theory to build on the previous one, so that lessons could be learned and theories explored and extended, without the inflexibility inherent in a more conventional survey.

The methodology was probably the only one which would have been capable of yielding the results achieved. Although quite time-consuming, due to the necessity to arrange, conduct, record, transcribe and analyse interviews, it is felt that no other methodology could have provided the depth of qualitative material that revealed not just a preferred world, but, on closer examination, a set of potential criteria behind this choice.

Grounded theory is open to many criticisms, as described in section 3.2.3, and must be seen as flawed in some degree - it is doubtful whether it can really be theory-neutral, there is a question about interviewees' intentionality, there may be some constructivism, and there are also questions regarding the validity of interpretation. However, in this study, the use of grounded theory techniques has opened up an area of user experience which would have been very difficult to explore using a quantitative methodology, which in itself would have fundamentally changed the relationship between researcher and users. Grounded theory may not be able to live up to all the claims made by its supporters, but it remains the best tool for an investigation of this type.

Without the iterative approach, the development from ideas to testing and back to ideas could not have worked. Without the free and unstructured approach, it seems less likely that the richness of the input could have been preserved, and without the interactive element provided by using interviews, rather than, for example, diaries, the opportunity was provided to explore topics at an appropriate level of detail.

There are many threads to follow through such an amount of primary material, and many of these threads appear in unexpected places, so that two or three distinct interviews may turn out to have quite close and complex relationships, when viewed from one perspective, yet seem almost completely unrelated from another. In this study, for example, the notion of "path" or "process" crosses the more structural or concrete versus abstract dimensions which are useful from another perspective. One of the rewards from this study has been an enhanced sensitivity to, and an increased respect for, the complex factors which might 
influence user-led design, and it is hoped that this may inform further research in the field.

\section{References}

Bryant, A. and Charmaz, K., eds. (2007) The Sage Handbook of Grounded Theory. London: Sage Publications.

Bryman, A. (1988) Quantity and quality in social research. London: Routledge.

Charmaz, K. (2006) Constructing grounded theory: a practical guide through qualitative analysis. Thousand Oaks, CA: Sage Publications.

Glaser, B. G. (1998) Doing grounded theory: issues and discussions. Mill Valley, CA. The Sociology Press.

Glaser, B.G. (2002) Constructivist grounded theory? Forum Qualitative Sozialforschung / Forum: Qualitative Social Research. 3(3). [online] URL: http://www.qualitative-research.net/fqs-texte/3-02/3-02glaser-e.htm [accessed 08.10.11].

Glaser, B. G. (2009) Jargonizing: the use of the grounded theory vocabulary. The Grounded Theory Review, vol.8, no.1, pp.1-15.

Glaser, B.G. and Strauss, A.L. (1967) The discovery of grounded theory: strategies for qualitative research. New York: Aldine de Gruyter.

Grounded Theory Institute (2012) URL: http://www.groundedtheory.com/ [accessed 30.05.12]

Lincoln, Y.S. and Guba, E.G. (1985) Naturalistic inquiry. London: Sage.

Linton, F. et al. (1999) Building user and expert models by longterm observation of application usage. In: J. KAY, ed.

Proceedings of the 7th International Conference on User Modeling. 20 - 24 June. New York: Springer-Verlag. pp. 129-138

Second Life. (2011) General discussion forum [online] URL: http://community.secondlife.com/t5/General-Discussion-Forum/Number-OfMembers/m-p/885357 [accessed 29.05.12]

Silverman, D. (2006) Interpreting qualitative data: methods for analysing talk, text and interaction. 3rd ed. London: Sage.

Sperber, D. and Wilson, D. (1995) Relevance: communication and cognition. 2nd ed. Oxford: Blackwell.

Strauss, A.L. and Corbin, J. (1990) Basics of qualitative research: grounded theory procedures and techniques.. Newbury Park, CA: Sage. 
Sutcliffe, A. (2003) Multimedia and virtual reality: designing multisensory user interfaces. Mahwah, NJ: Lawrence Erlbaum.

WWW Virtual Library (2011) URL: http://vlib.org/ [accessed 08.10.11].

\section{Open access and copyright}

Library and Information Research is an open access journal. A freely available copy of this paper may be downloaded from the journal's website: http://www.cilipjournals.org.uk/lir

Copyright and associated moral rights in works published in Library and Information Research are retained by the author(s) but this paper may be used freely, with proper attribution, in educational and other non-commercial settings. 